\title{
Domino paired kidney donation: a strategy to make best use of live non-directed donation
}

Robert A Montgomery, Sommer E Gentry, William H Marks, Daniel S Warren, Janet Hiller, Julie Houp, Andrea A Zachary, J Keith Melancon, Warren R Maley, Hamid Rabb, Christopher Simpkins, and Dorry L Segev

\section{Current models for allocation of kidneys from living non-directed donors}

Living non-directed (LND) donors, also known as altruistic, good Samaritan, anonymous, or benevolent community donors, are a new and rapidly growing source of solid organs for transplantation. ${ }^{1-6}$ The willingness of individuals to donate organs without a designated recipient has been unexpected, but has probably developed as a societal response to the growing crisis in organ availability. In the context of this shortage, health professionals have attempted to make the best use of kidneys from LND donors. We present a novel application of paired donation that has the potential to multiply the number of recipients who can benefit from each LND donation.

At present, there is no universally accepted system for allocation of organs from LND donors. Selection of recipients has been at the discretion of the transplant centres where LND donors have presented and has generally been guided by one of three models: donorcentric, recipient-centric, or sociocentric allocation. ${ }^{7}$ Each of these models is supported by valid ethical arguments. ${ }^{7-12}$

The main goal of donor-centric allocation is to ensure a successful outcome for the recipient. A good outcome provides justification for medical professionals to assist a person who is not ill to put themselves in harm's way to aid another. A positive result also gives an LND donor a sense that their effort was fruitful and worthwhile. However, this model dictates allocation to the healthiest patient on the transplant waiting list. These recipients are the most likely to have good outcomes on dialysis or with organs from deceased donors, and therefore are arguably the least in need.

Recipient-centric allocation is based on the belief that society has a responsibility to protect its most vulnerable and disadvantaged members. Under this model, organs from LND donors are given to those patients in the greatest need, those for whom a kidney transplant might be truly life saving, or those disadvantaged under the existing system for allocation of kidneys from deceased donors. This model mainly benefits children, patients who have no vascular access, highly sensitised patients, and those with life-threatening medical illnesses related to dialysis. However, because the recipient-centric model accords priority to such patients, it tends to yield unacceptably poor transplant outcomes, and could lead to a negative public perception of LND donation.

Under the third model, of sociocentric allocation, the LND donated organ is treated as a public resource that should be allocated in the fairest and most equitable way, irrespective of outcome or need. This rationale dictates that the recipient should be the patient at the top of the transplant waiting list administered by the United Network of Organ Sharing (UNOS). UNOS oversees the allocation of deceased donor organs in the USA, using a so-called match run algorithm that ranks potential recipients according to agreed criteria. The limitations of this model are that a patient at the top of the list will probably receive a kidney from a deceased donor in the near future, and that they will have already incurred the costs, and exposure to comorbidity, that result from a long period on dialysis.

The waiting list for deceased donor kidneys can be circumvented by patients who find a willing live donor. But direct donation might be complicated by differences in blood type and by HLA sensitivity. Some incompatible donor-recipient pairs enter into programmes that facilitate paired donation, also known as kidney paired donation. A donor and recipient who have incompatible blood groups or HLA sensitivity can be matched with another incompatible pair, to result in two compatible transplants (figure). ${ }^{13-19}$ Although there are many ways to match up a pool of incompatible pairs, the mathematical technique of optimisation helps to find out which matches will yield the best results. ${ }^{13}$ Nevertheless, even in paired-donation programmes in which mathematical optimisation is applied, more than $50 \%$ of the incompatible pairs in the pool remain unmatched. ${ }^{13,20}$ In many cases, pools of incompatible donor-recipient pairs have a high proportion of patients with blood types that are hard to match and those with HLA sensitisation.

\section{Domino paired donation}

In this study, we examine the effect of allocating a LND donor organ to a pool of incompatible donor-patient pairs. This strategy allows a new type of paired donation, which we call domino paired donation, in which the LND donor's gift initiates a chain of matches. First, the LND donor's kidney is matched to a recipient who has a willing but incompatible donor (figure and panel). The recipient's incompatible donor can, in turn, agree to give a kidney to the next compatible patient on the transplant waiting list, producing a domino effect. In this way, two live donor kidney transplants result, and the LND donor's gift is multiplied.

We have also developed a mathematical simulation to quantify the potential benefit of allocation of LND donors' kidneys by use of the domino paired donation model. Since the first LND donation was reported to UNOS in
Lancet 2006; 368: 419-21 See Comment page 346

Department of Surgery (R A Montgomery MD, SE Gentry PhD, D S Warren PhD J Hiller RN, J K Melancon MD, W R Maley MD, C Simpkins MD, D L Segev MD) and Department of Medicine (J Houp, Prof A A Zachary PhD H Rabb MD), Johns Hopkins University School of Medicine, Baltimore, MD, USA;

Department of Mathematics, United States Naval Academy, Annapolis, MD, USA (SE Gentry); and Department of Organ Transplantation, Swedish Medical Center, Seattle, WA, USA (W H Marks MD)

Correspondence to: Dr Robert A Montgomery rmonty@jhmi.edu 


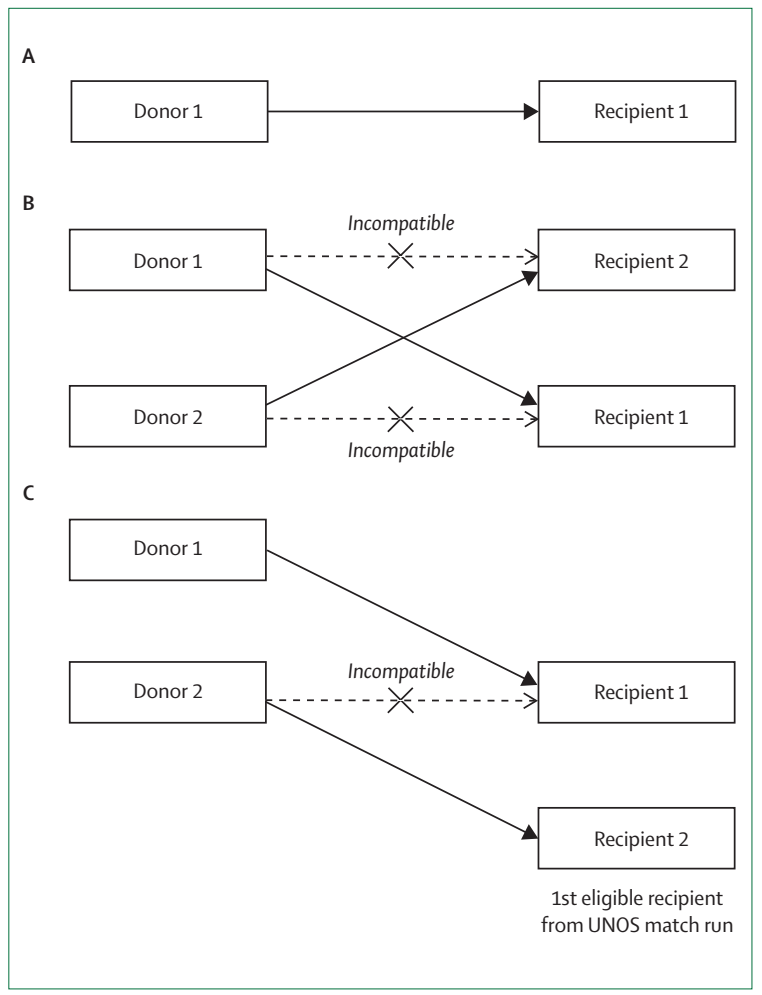

Figure: Strategies to expand the use of kidneys from live donors (A) Current one-to-one model. (B) Conventional paired donation between two incompatible donor-recipient pairs. (C) Proposed model of domino paired donation

1998, this type of kidney transplant has steadily risen in frequency, with a total of 302 procedures reported in the USA to date. ${ }^{1}$ We used the optimised algorithm we have previously reported ${ }^{13}$ to simulate paired donation by finding reciprocal matches within pools of incompatible donor-recipient pairs. We then compared the results with the numbers of simulated transplants obtained if LND donors were introduced to these pools by use of domino paired donation. On the basis of these comparisons, we estimate that if domino paired donation had been adopted in the USA since the first LND donation, 583 transplants would have been accomplished, rather than 302. The number is slightly less than two transplants per LND donation because not every LND donor will find a match in a pool of incompatible pairs. (Where a domino paired donation cannot be arranged for a LND donor, they can be paired with the next compatible patient on the transplant waiting list by use of a UNOS match run.) Three-way domino paired donation is also possible, and can further increase the benefit derived from each LND donor.

\section{The ethics of domino paired donation}

The domino paired donation model would satisfy the ethical tenets of the three main philosophies that are commonly used to guide allocation, and for this reason it could be predicted to gain wide acceptance. The donor-
Panel: A clinical example of domino paired donation

We obtained approval for our protocol from the Johns Hopkins University Ethics Committee and Legal Office. ${ }^{14}$ Among the ethical arguments for domino paired donation, the Ethics Committee considered the human consequences of the present shortage of donors and the autonomy of the informed individual to make decisions about their wellbeing to be particularly compelling justifications. Each participant was medically cleared and gave informed consent after counselling about untoward events that might prevent the completion or success of a transplant. All operations were done simultaneously, and anonymity was maintained throughout.

We trialled a domino paired donation in which a 28 -yearold LND donor (D1), who had blood type 0 , gave her kidney to a 48-year-old recipient (R1) with end-stage renal disease from IgA nephropathy. He was in the paired donation pool because he and his 51-year-old wife, D2, had incompatible blood types. R1 was blood type B with strong antibody reactivity to the A blood-group antigen, and his wife (D2) was blood group A1. D2 agreed to donate to a patient on the transplant waiting list. According to standard procedure, a UNOS match run was done for blood type A. The first eligible patient on the transplant waiting list was a 48-year-old woman (R2) with renal failure from polycystic kidney disease. R2 had been waiting on the list for a kidney from a deceased donor for 2 years. Domino paired donation took place as shown in figure 1: R2 received a kidney from D2 at the same time that R1 received a kidney from the LND donor (D1) Both recipients were shown to have serum creatinine concentrations of $124 \mu \mathrm{mol} / \mathrm{L}$. The reference range for serum creatinine is $45-140 \mu \mathrm{mol} / \mathrm{L}$.

centric model would be served because donor paired donation would increase the likelihood of a good outcome, by spreading the risk of recipient graft loss across more people. That is, even if the transplant of the LND donor's kidney was unsuccessful, domino paired donation would enable a second chance of a positive result, when the paired donor's kidney was given to a recipient on the waiting list. According to the recipientcentric model, priority should go to recipients with the greatest need or disadvantage. In many cases, incompatible donor-recipient pools have a high proportion of patients with blood types that are hard to match, and those with HLA sensitisation. Therefore, the domino paired donation model would help these patients, who are disadvantaged by the current allocation system because they tend to wait longer for a kidney and are more likely to develop the comorbid disorders associated with prolonged dialysis. The sociocentric allocation model aims to achieve fairness and equity by allocating kidneys to patients at the top of the waiting list. The domino paired donation model would conform to this standard, since the paired donor's kidney would be 
allocated to the next compatible patient on the UNOS registry.

\section{Conclusion}

At a time of growing crisis in organ availability, this study shows that the current system of allocating LND donor organs does not achieve the greatest possible benefit from this new and growing source of kidneys. Implementation of domino paired donation on a national or regional scale should improve consistency and fairness across transplant centres, but would reduce each transplant centre's autonomy in making decisions about allocation of organs. Domino paired donation would also be affected by the same practical limitations as paired donation in terms of the logistical difficulty of arranging paired donations between diverse and distant institutions. Although in our limited experience LND donors have welcomed this allocation model, we would need to better understand the attitudes of LND donors, paired donors, and the public before domino paired donation is adopted as a standard policy. However, our study shows that the use of domino paired donation has the potential to increase both the quantitative and qualitative benefit of each LND donation.

Role of the funding source

No funding source had a role in study design, data collection, data analysis, data interpretation, writing of the report, or the decision to submit the paper for publication. The corresponding author had full access to all of the data in the study and had final responsibility for the decision to submit for publication.

\section{Conflict of interest statement}

We declare that we have no conflict of interest.

\section{References}

1 United Network for Organ Sharing. Organ procurement and transplantation network data. http://www.unos.org (accessed May 24, 2005).

2 Henderson AJ, Landolt MA, McDonald MF, et al. The living anonymous kidney donor: lunatic or saint? Am J Transplant 2003; 3: 203-13.

3 Gilbert JC, Brigham L, Batty DS Jr, Veatch RM. The nondirected living donor program: a model for cooperative donation, recovery and allocation of living donor kidneys. Am J Transplant 2005; 5: 167-74.
4 Gohh RY, Morrissey PE, Madras PN, Monaco AP. Controversies in organ donation: the altruistic living donor. Nephrol Dial Transplant 2001; 16: 619-21.

5 Jacobs CL, Roman D, Garvey C, Kahn J, Matas AJ. Twenty-two nondirected kidney donors: an update on a single center's experience. Am J Transplant 2004; 4: 1110-16.

6 Matas AJ, Garvey CA, Jacobs CL, Kahn JP. Nondirected donation of kidneys from living donors. $N$ Engl J Med 2000; 343: 433-36.

7 Marks WH. Towards understanding living non-directed donation. In: Symposium on living non-directed donation; October 27-29, 2004; Seattle, WA, USA. http://www.nondirecteddonor.org (accessed June 27, 2005).

8 Adams PL, Cohen DJ, Danovitch GM, et al. The nondirected livekidney donor: ethical considerations and practice guidelines: A national conference report. Transplantation 2002; 74: 582-89.

9 Landolt MA, Henderson AJ, Barrable WM, et al. Living anonymous kidney donation: what does the public think? Transplantation 2001; 71: 1690-96.

10 Ross LF, Glannon W, Josephson MA, Thistlethwaite JR Jr. Should all living donors be treated equally? Transplantation 2002; 74: 418-22.

11 Spital A. Should people who donate a kidney to a stranger be permitted to choose their recipients? Views of the United States public. Transplantation 2003; 76: 1252-56.

12 Spital A. Public attitudes toward kidney donation by friends and altruistic strangers in the United States. Transplantation 2001; 71: 1061-64.

13 Segev DL, Gentry SE, Warren DS, Reeb B, Montgomery RA. Kidney paired donation and optimizing the use of live donor organs. JAMA 2005; 293: 1883-90.

14 Montgomery RA, Zachary AA, Ratner LE, et al. Clinical results from transplanting incompatible live kidney donor/recipient pairs using kidney paired donation. JAMA 2005; 294: 1655-63.

15 Ross LF, Rubin DT, Siegler M, Josephson MA, Thistlethwaite JR Jr, Woodle ES. Ethics of a paired-kidney-exchange program. N Engl J Med 1997; 336: 1752-55.

16 Delmonico FL. Exchanging kidneys-advances in living-donor transplantation. N Engl J Med 2004; 350: 1812-14.

17 Delmonico FL, Morrissey PE, Lipkowitz GS, et al. Donor kidney exchanges. Am J Transplant 2004; 4: 1628-34.

18 de Klerk M, Keizer KM, Claas FH, Witvliet M, Haase-Kromwijk BJ, Weimar W. The Dutch national living donor kidney exchange program. Am J Transplant 2005; 5: 2302-05.

19 Park K, Moon JI, Kim SI, Kim YS. Exchange donor program in kidney transplantation. Transplantation 1999; 67: 336-38.

20 Segev DL, Gentry SE, Melancon JK, Montgomery RA Characterization of waiting times in a simulation of kidney paired donation. Am J Transplant 2005; 5: 2448-55. 